\title{
Ueber einige Voraussetzungen beim Beweise des Dirichlet'schen Principes.*)
}

Von $\mathrm{E}$. Heine in Halle.

Es sollen hier einige Voraussetzungen und Schlüsse geprïft werden, auf denen, nach den vorliegenden Mittheilungen, der Beweis des Dirichlet'schen Principes in der Lehre vom Potentiale beruht.

1) Die erste Foraussetzung besteht in Folgendem: Die auf der Begrenzung des Raumes $t$, welche eine geschlossene Fläche bildet, gegebene continuirliche und einwerthige function kann in das Innere and ebenso in den äusseren Raum, wenigstens auf eine Art, derartig fortgesetzt werden, dass die Fortsetzung dieselben Bedingungen der Stetigkeit und Endlichkeit erfüllt, wie das Potential der Vertheilung einer Masse auf der Oberfläche.

Die im Folgenden nicht erklärten Bezejchnungen sind dieselben wie bei Gauss in dem Werke: Allgemeine Lehrsätze in Beziehung auf die im verkehrten Verhältnisse des Quadrates der Entfernung wirkenden Anziehungs - und Abstossungskräfte; ferner wird, wie üblich,

durch $\Delta V$ bezeichnet.

$$
\frac{\partial^{2} V}{\hat{\partial} x^{2}}+\frac{\hat{\partial}^{2} V}{\hat{\partial} y^{2}}+\frac{\hat{o}^{2} V}{\hat{\partial} z^{2}}
$$

Die Continuitätsbedingungen, welche, wie man voraussetat, wenigstens eine Fortsetzung $V$ erfüllt, bestehen also darin, dass $V$ im ganzen Raume stetig ist, $\frac{\partial V}{\partial x}, \frac{\partial V}{\partial y}$ und $\frac{\partial V}{\partial z}$ nur bis zur Grenzfläche, sowohl im äusseren als im inneren Ranme. (Beweisen will man bekanntlich, dass es auch eine Fortsetzung $V_{1}$ giebt, für welche noch ausserdem $\Delta V_{\mathrm{g}}=0$.)

Dirichlet selbst benutzt das Princip bei der Theorie des Potentiales zum Beweise desjenigen Satzes, mit dem Gauss seine oben genannte Arbeit krönt (Nr. 36.), nach welchem eine Massenvertheilung in einem körperlichen Raume sich durch eine Belegung der Oberfläche

*) Aus den Nachrichten der Göttinger Gesellschaft der Wissenschaften vom 16. August 1871, mit Zusätzen des Verfassers. 
mit Masse ersetzen lasst. Die an der Obertuche gegebene Function ist bei diesern Beweise nieht vollig allgemein; sie ist uämlich gleich dem Werthe, welchen das Potential $\int \frac{k d t}{r}$ der gegebenen, im körperlichen Raume vertheilten Masse an der Oberfïche annimut. Sie kann also wirklich, den Bedingungen gemüss, fortgesetct werden, nünlich durch dieses Potential selbst.

Cm dann nachzuweisen, dass die gesamante Masse zor Belegung verwandt werden kann, muss mau die Fortsetzung auch noch für den Fall bilden, dass die für die Oberfäche gegebene function coustant, $=1$ ist. Durch $V=1$ lasst sich diese Function in den inneren Raum fortsetzen, aber nicht durch denselben Werth in den äusseren Raum, wegen der Bedingung der Lndlichkeit $(x V$ soll endlich bleben), Nach den Principien, welche jch an Schlusse einer brieflichen Mittheilung über Variationsrechnong in den mathematischen Annalen Bd. 1, S. 191 andeutete, finde ich diese Fortsetzung, indem ich um den Anfangspunt als Mittelpunkt eine Kngel mit dem liadius a beschreibe, welche den Raum $t$ ganz einschliesst. Beucichnet $p$ die Entfernong eines beliebigen l'unktes in Raume vom Anfangspunkte, so kaun man als Fartsetzung folgende Function $\boldsymbol{V}$ betrachten: Von der Begrenzung des Körpers $t$ bis $\varrho=a$ sei $V=1$; von $\varphi=\alpha$ bis $\varrho=\infty$ sei:

$$
V=1-\left(\frac{e-\alpha}{e}\right)^{3}
$$

Diese einwerthige Functian genügt allen Bediagungen in äasseren Raume, wenugleich sie daselbst nicht in ganzen Verlaufe durch ein und dasselbe analytische Geset dargestellt wird. (Kine solche forderung ist aber auch gar nicht gestellt.) In der That sind quch thre Differentialquotienten bis zu den zweiteu incl, nach $x, y$, ebento wie die Function selbst, einwerthig and stetig, was man devtlich einsieht, wenn man an den Uebergangsstellen auf die Definition des Differentialquotienten zurüukgeht.

Wegen der Wichtigkeit der von C. Neunann mit dem Namen der Green'schen belegten Function will ich noch zeigen, dass die in Rede stehende Vorbedingung der Existenz auch für sic ortillt werden kann, wie auch $t$ beschaffen ist, wenn nur die Begrenzung den Bedingungren der Nr. 16. bei Gauss genügt.

Sei daan $A$ ein gegebener fester Pankt innorluall $t$ oder ansserkalb: $P$ bezeichne die Punkte im Rauane, $P_{0}$ an der begrenzenden Flähe; es sei $A P=r, A P_{p}=r_{1}$. Es soll die Function, welche an der Oberfache $\frac{1}{r_{0}}$ ist, unseren Bedingungen gemäss fortgesetzt werden. Dies hat nur für den inneren resp. äusstron Ranm Schwierigkeiten, 
da für die Punkte $\mathcal{P}$ des äusseren resp, inneren Raumes $\frac{1}{r}$ eine braụchbare Fortsetzung ist. Legt man nun um $A$ als Mittelpunkt eine Kugel mit einem beliebigen Radius $\alpha$, die aber ganz innerhalb, resp. gan\% ausserhalb des Raumes $t$ liegt, und bezeichnet mit $U$ im Inneren und auf der Kugel đie Grösse 1, von der Kugeloberfläche bis zur Begrenzung von $t$ aber Null, so wird

$$
V=\frac{1}{r}+\frac{\left(r^{6}-\alpha^{6}\right)^{3}}{r \cdot \alpha^{18}} \cdot U
$$

eine Fortsetzang in den inneren resp. den äusseren Raum, welche allen Bedingungen der Stetigkeit und Endlichkeit genügt.

Abgesehen von den Fällen, in denen mehrere geschlossene Flächen auftreten, und die ich durch die gleichen Pribcipien erledigen kann*), hat Dirichlet anch in den Anwendungen auf Elektrostatik nur solche Functionen wie die hier besprochenen von der Oberfiäche ins Innere fortzusetzen. Die Richtigleit der ersten Annahme in den bei ihm vorkommonden Füllen ist daher nachgewiesen.

2) Es wird ferner vorausgesetet, dass es mehr als eine, und alaher unendlich viele, den gleichen Bedingungen wie oben genügende Fortsetzungen giebt. Ist eine erste Fortsetzang $V$, so lisst sich offenbar jede andere als $V+Z$ darstellen, wo $Z$ alle, den gleichen Bedingungungen der Endlichkeit und Stetigkeit genügende Fortsetzungen der für die Oberfïale gregebenen Function Null bezeichnet.

Solcher $Z$ giebt es immer unendlich viele. Ist nämlich $\varphi(x, y, z)=0$ irgend eine beliebige geschlossene algebraische Fläche, z. B. eine $\mathbf{K u}$ gel, die ganz innerhalb oder ganz ausserhalb $t$ liegt (es sei $\varphi$ eine ganze Function von $x, y, z)$; ist ferner $D$ irgend eine mit ihren ersten beiden Difierentialquotienten innerhalb des ron $\varphi=0$ umschlossenen Raumes einwerthige, stetige und endliche Function, ausserbalb desselben aber 0 , so wird,

$$
W=\varphi^{3} U
$$

gesetzt, $Z=W$ immer eine von den Functionen $Z$ seill. Es ist klar, dass unendlich viele $U$, selbst bei festgehaltenem $\varphi$, existiren; eine Function $U$ findet man schon, wenn man im Innern des durch $\varphi=0$ begrenaten Raumes $U=1$ setzt. Um noch andere zu erhalten, denke man sich z. B. diesen Körperraum irgendwie continuirlich und gleichartig rit Masse erfullt, und kann dann für $U$ in jedem Punkte des Inneren das Potential dieser fingirten Masse in demselben Punkte nehmen.

*) Die weitere Austihurung habe ich diesem Abdrucke als Anhang hinzu- 
Die zweite Voraussetzung ist daher in donsellen Fallew wie die erste berechtigt.

3) Es folgt nun bei Dirichlet eine Annahme, auf welche ich hier nicht näher eingehe, und auf die ich bei einer anderen Gelegenheit zurückzukommen denke, dass es näwlich eine oder einige Fortsetzungen in den inneren Raum giebt - Aehnliches gilt fur den äusseren Raum; hier wird der Kürze balber nur der innere betrachtet -welche

$$
\int\left(\left(\frac{c V}{\hat{c} x}\right)^{2}+\left(\frac{\hat{c} V}{\hat{c} y}\right)^{2}+\left(\frac{\hat{c} V}{c z}\right)^{2}\right) d t
$$

zu einem Minimum machen. Aus dieser Annahme ergiebt sich, wenigstens eine von diesen Fortsetzungen, $V_{1}$, müsse so beschaffen sein, dass für jede Function $Z$ (m. 5. Nr. 2.) das Integral

$$
\int Z \Delta V_{1} d t
$$

verschwindet. Hicraus will man selliessen, im hanme $t$ müsa $\Delta V_{\text {, }}$ im allgemeinen $N_{u l l}$ sein, d. L. mit Ausuabme böchstens von Pantiten, Linien und klächen. Dieser Schluss soll hikr gcprïft worden.

Er ist, in Folge des Vorhergehenden, fär jedes Stück des Raumes $t$ erlanbt, in dem die Function $\Delta V_{1}$ inr Zeichen nicht unendich oft ändert. Man kann dann nämlich die Stüeke, in welchen $\Delta V_{3}$ das gleiche Zeichen behall, beliebig nabe durch Körper mit algebraischer Begrenzung $\varphi=0$, x. B. mit Kugeln ausfüllen und für $Z$ eive Fanction $W$ aus Nr. 2, wahlen, die in dem Raume, in welebem sie nieht verschwindet, ihr Zejchen nicht wechselt, sodass das Interral sich allein auf den Theil bezieht, der von einer Flüche $\Psi=0$ eingeschlossen ist und in weichem daher $W \Delta V_{1}$ sein Zeichen nicht wechselt, woraus folgt, dass $\Delta V_{1}$ in Allgemeinen Null ist.

In allen Fälen, die in $\mathrm{N}_{\mathrm{r}}$. 1. erwähnt sind, kann man annehmen, dass $\Delta V_{t}$ sein Zeichen im Allgeneinen nicht unendlich oft ändert. Die eine Fortsetzung $V$ der an der Oberfläche gegobensen Function aus Nx. 1., die sich auf die Green'sche Function bezielat, nâmolich

$$
V=\frac{1}{r}+\frac{\left.r^{6}-\alpha^{6}\right\}^{3}}{r \cdot a^{6}} \cdot U_{3}
$$

besitzt diese Eigenschaft augrenscheinlich, cbenso wit die andere Func. tion, welche man dort findet, vämlich:

$$
V=1-\left(\frac{e-s}{e}\right)^{s}
$$

und ebenso wie das Körperpotential in Nr. 1.

$$
V=\int \frac{x_{i} d t}{r},
$$


vorausgesetzt, lass die Eigenschaft in dem letzteren Falle der Dichtigtigkeit $t$ der zu vertheilenden Masse, wo diese unstetig sein sollte, selbst zukommt. Die Masse darf daher z. B. keine magnetische sein, die aber bereits durch die F'estsetzungen von $G$ auss über die Dichtigkeit in Nr. 9. ausgeschlossen ist und selbst nach Aufhebung einiger Beschränkungen in Nr. 11. noch ausgeschlossen bleibt. Man kann freilich die Betrachtung von Potentialen magnetischer Massen, nachdem man sie durch Zusammenfassen von je zwei Gliedern der Summe $\Sigma \frac{\mu}{r}$ (s. Nr. 2. bei Gauss) in ein Integral verwandelt hat, auf die der Potentiale von Massen mit continuirlicher Dichtigkeit zurückführen.

Ist also diese Art von Massen, auf welche die Untersuchungen von Gauss nicht überall anwendbar sein würden, ausgeschlossen, so besitzt $\Delta V$ in Nr. 1. die für $\Delta V_{1}$ geforderte Eigenschaft in Bezug auf die Zeichenwechsel. Unter den Functionen $W$ in $\mathrm{Nr}$. 2. giebt es offenbar unendlich viele von solcher Beschaffenheit, dass $V+W$ dieselbe Eigenschaft besitzt. Stellt nun $V_{1}$ nicht das Minimum unter allen Fortsetzungen, sondern nur unter denen vor, welche die erwähnte Eigenschaft in Bezug anf die Zeichen besitzen, und deren es unendlich viele giebt, so ist für dieses $V_{1}$ demnach der Schluss, dass $\Delta \nabla_{1}$ in Allgemeinen Null sei, berechtigt.

\section{A $\mathrm{n} \mathrm{h}$ a $\mathrm{n}$.}

(S. S. 628.)

Es mögen $n$ Flächen vorliegen, die nicht untereinander zusammenhängen, oder, genauer ausgedrückt, es sollen alle Punkte einer jeden von den Punkten der übrigen um mehr als eine angebbare feste, wenn auch noch so kleine Grösse entfernt sein.

Die Aufgabe, eine Function $W$ zo bilden, welche auf jeder von diesen Flächen gegebene Werthe annimmt, lässt sich auf n einfachere reduciren. Man hat nur nöthig, $n$ Functionen za bilden, von denen die $\nu^{\text {te }}$ - wenn $v$ successive die Zahlen 1, 2, etc., $n$, vorstellt - anf der $v^{\text {ten }}$ Fläche die gegebenen Werthe annimmt, auf den übrigen $n-1$ Flächen sich aber in Null verwandelt. Die Summe solcher $n$ Functionen giebt eine Function $W$ von der verlangten Beschaffenheit.

Ist nuu $v$ irgend eine Function, die an $n-1$ gegebenen Flächen gegebene Werthe anninmt, so werde ich aus derselben eine Function $\nabla$ bilden, welche mit $v$ an den $n-1$ Flächen übereinstimmt, an einer 
gegebenen $n^{\text {ten }}$ Fläche aber überall verschuoindet. Genügt $v$ den Bedingungen der Stetigkeil und Eudlichkeit, so wird auch dieses $\boldsymbol{V}$ ihnen genügen.

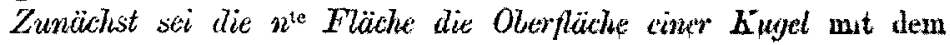
Radius $\alpha$ und dern Mittelpunkte $A$; im Inneren der Fugel moge lein Theil von einer der anderen Flächen liegen. Man beschreitu um $A$ eine zweite Kugel mit einem Radius $\beta$, der grösser als $\alpha$, aber noch so klein ist, dass auch diese Kugel kein Stück vou einer der ubrigen $n-1$ Flächen einschliesst. Ist $r$ die Entfernung eines beliebigen Punktes $P$ von $A$, so setze ich $F$ in den Punkten $P$, die

ausserhalb oder auf der Kugel mit dem Radius $\beta$ liegen, gleich $v$; auf und in der von den beiden Kugelfächen begrenzten Schale gleich

$$
v\left[1-\left(\frac{\beta^{2}-r}{\beta^{2}-\alpha^{2}}\right)^{3}\right]^{3}
$$

drittens, innerhalb der Kugel nit dem Radius a gleich Null.

Diese Function $\nabla$ genügt den Bediagungen der Sietigkeit und Endlichkeit, vorausgesetzt, dass $v$ ihnen genügt, erhält an dep $n-1$ Flächen dieselben Werthe wie $v$, und verwandelt sich sogar inuerhalb der ganzen Kugel mit dem Radius $\alpha$ in Noll.

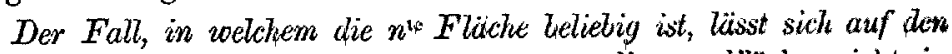
vorigen speciellen zurüclführen. Kann man die $n^{\text {ts }}$ Flüche nicht in eine einzige Kugel einschliessen, die kein Stück einer von den $n-1$ anderen Flächen enthält, so erfolgt die Einschliessung durch sine grössere aber endliche Zahl $s$ van Kugeln, die nach ibren Mittelpunkten $A_{1}, A_{2}$, etc., $A_{x}$ heissen mögen. Man lege diese Kugeln so, dass jede ein Stück der $n^{\text {tea }}$ Obertiäche, aber keines yon den übrigen $n-1$
Flächen enthält; die Möglichleelt, auf diese Art durch eine endiche Anzahl von Kugeln alle Theile der $n^{\text {len }}$ Fläche einzuschliessen, ist klar, da ein und dasselbe Stück in rnebreren Kugel vorkommen darf.

Nach diesen Vorbereitungen bilde man aus $v$, nach der im speciellen Falle angewandten Methode, eine Function, wie die oben $\boldsymbol{V}$ genannie, die nun $v_{1}$ heisse, welche nieht nur an der Oberfiäche dex Kugel $A_{1}$, sondern auch im Inneren derselben Null ist und an den übrigen $n-1$ Flächen mit $v$ übereinstimmt. Bildet man aus $v_{1}$ nach derselben Methode eine Function $v_{2}$, die auch in der Kugel $A_{2}$ verschwindet etc., endlich aus $v_{s-1}$ eine Function $v_{s}$, die noch in $A_{*}$ versehwindet, so wird $v$, aq den gegebenen $x-1$ Flächen dieselben Werthe wie $v$ besitzen, und an der $n^{\text {tea }}$ wie verlangt wurde, verschwinden; also wird $v_{s}$ die Aufgabe lösen.

Indem man $n$ successive die Werthe 2,3 , etc. bis zu einer belicbigen ganzen Zahl beilegt, erhält man hieraus den Satz: 
Kann eine Function, die auf einer einzigen Fläche gegebene Werthe besitzt, nach den Bedingungen der Stetigheit wnd Endlichkeit in den Raum fortyesetzt werden, so lässt sie sich auch so fortsetzen, dass sie noch ausserden die Bedingung erfullt, auf einer beliebigen Anzahl gegebener Flächen, welche mit der ersten nicht zusammenhängen, zu verschuinden.

Halle, September 1871. 